\title{
An Improved Nonlinear Multi-Objective Optimization Problem Based on Genetic Algorithm
}

\author{
Yali Yun and Yaping Li* \\ College of Computer and Information Engineering, Luoyang Institute of Science \\ and Technology, Luoyang 471023, China. \\ E-mail:jsjxexam@163.com
}

\begin{abstract}
Genetic algorithms for multi-objective optimization problem to be solved were studied. Through the elitist strategy analysis, it is an improved multi-objective optimization algorithm. The algorithm uses a data warehouse to store the optimal solution produced by individuals in each generation, from the way individuals adopt measures to phase out the individual data warehouse identical or similar, the algorithm also improved selection operator, so that the algorithm adaptive capacity enhancement, the new algorithm improves the algorithm performance, improves the quality of understanding between sets, can get a lot of optimal and balanced.
\end{abstract}

Keywords: genetic algorithm; multi-objective optimization; optimal solutions; crossover; mutation

\section{Introduction}

Multi-objective optimization is a rapidly developed disciplines, is an important branch of optimization, which in some sense the main research goals while multiple numerical optimization problem attracted many scholars. In real life, the natural human transformation program planning and design process in general reflect the "maximize efficiency, minimize costs," the basic principle of optimization measures in co-optimal strategies for solving the problem of how to get the win goals, in the non-cooperative game problem of how to make your own interests to maximize, minimize the other's benefit, and control engineering steady, accurate, and fast timedomain index and degree of stability region, the system bandwidth frequency domain characteristics comprehensive problems are actually multi-objective optimization problem, so multi-objective optimization problem can be seen everywhere in the real world. While the classical approach solves some optimization problem, but for multi-objective optimization problem but no efficient and practical solution, but many originated in the actual design of complex systems, modeling and planning problems, such as industrial manufacturing, capital operations, urban transport, forest management, reservoir design, the new urban layout and landscaping, energy distribution, etc., almost every important decision problems in real life to be in considering various constraints while optimizing a number of goals, and these problems involved in the plurality of target does not exist independently, are often coupled together and in a state of competition, each target has different dimensions and physical meaning, their complexity and competition optimization makes it very difficult. Therefore, the multi-objective optimization problem with a very important practical research meaning has become an attractive area of research.

In real life practical applications to solve practical optimization problems, if only to consider a goal, which we call single-objective optimization problem (Singleobjective optimization problem, SOP), otherwise termed A multi-objective optimization problem (Multi-objective optimization problem, MOP). Multi- 
objective optimization is an important field of optimization research direction, because a lot of scientific research and engineering practice optimization problems can be attributed to a multi-objective optimization problem [1]. These systems are located in the areas of industrial manufacturing, urban transport, capital budgeting, reservoir management, energy distribution, logistics, network communication, etc., can be said that there are multi-objective optimization problem everywhere.

\section{Related Works}

Multi-objective optimization ideas germinated in 1772, when Franklin proposed the multi-objective - how to reconcile the conflicting issues [2]. 1896, V.Pareto first is the economic balance of the study presented in multi-objective optimization problem, the introduction of the concept known as the Pareto optimal [3]. 1947, J.VonNeuman and O.Morgenstem in game theory mentioned in the writings of multi-objective decision-making issues, from the perspective of game theory laid the basis of the theory of economic behavior, causing people for multi-objective optimization research attention. 1951, TCKoopmans efficient solution introduced some basic definitions and results obtained for the multi-objective optimization discipline laid the initial foundation. At the same time, Kulm, who gives a Vector Extremism Problems necessary condition for an effective solution . 1953 Arrow and others have also put forward the concept of the effective point . Thus, the multiobjective optimization is attentions. From the late 1950s to the late 1960s , Chame, Karlin, Zadeh, Klingcr, Polak, Kceney and Geoffrion, who has made a more influential work, there has been weighted sum method, goal programming, $\varepsilon$ constraint method such as weight-based multi-objective optimization methods traditionally, during multi-objective optimization methods and theories of attention.

In recent years, with the evolutionary algorithm (Evolutionary Computation) technology and swarm intelligence (Swarm-Intelligence) method as well as the rise of research and practice in a wide range of applications, the development of multiobjective optimization techniques more rapidly, has become a hot field of study. About the same time the emergence of many multi-objective evolutionary Algorithms conference, the current evolutionary algorithm research has become in recent years, information science [4], artificial intelligence and computer science [5], one of the two major research focuses.

The traditional mathematical programming and simulated annealing algorithm is characterized by a single point serial search algorithm, can not use the concept of optimal solution Parco assess " the generation of evolutionary algorithms to solve the above problem provides a new idea " evolutionary algorithm search solution one of the biggest feature is that it is a group of search algorithm, it is natural to solve the above conventional multi- objective optimization algorithm parallelization difficult problem, effective use Parrot optimal solution becomes very reasonable, " and, evolutionary algorithm Pareto sets such as shape continuity and other properties are not sensitive to these two points, but often encountered in practical work, and use traditional mathematical methods are difficult to deal with " 1967 Rosenberg in his doctoral thesis was proposed based on available genetic search algorithm to solve multi-objective optimization problem ; but until 1985 there has been a genetic algorithm based on vector assessment (EvGA) [6], which is the first multi-objective evolutionary algorithm (MOEA): but until the 1990s , evolution multi-objective algorithm gradually become a hot research of many scholars, " so these algorithms can be roughly divided into [7] : non-Pareto solution set based on the concept of algorithm, based on the concept of Pareto set algorithm and improved algorithm . non-Pareto techniques including : polymerization method, VEGA algorithm lexicographic method, G-constraint method, the target vector method ; 
In the algorithm design, Yu-Ping Wang [8], respectively, the orthogonal design, uniform design combined with genetic algorithm for solving multi-objective optimization gives the new method. [9] And so introduces a $\varepsilon$ - constraint method Augmented Lagrange algorithm for multi-target collaboration. [10] And so on the current trends in multi-objective optimization studies are summarized and presented themselves to the further development of multi-objective optimization of view.[11] demonstrated that the method can be used for quick sort groups of individuals classified, also discussed using clustering methods to maintain the diversity of population.

\section{Mathematical Models of Genetic Algorithms}

In practical applications, it is often encountered in multi-criteria or objectives, design and decision-making problems, such as securities investment issues, investors in order to get higher returns, you need to select the best stocks to invest in, in general, a outstanding shares have the following characteristics: good performance, low price-earnings ratio, growth higher, but usually these goals are in conflict, such as the current domestic steel industry generally better performance of listed companies, earnings are relatively low, but the steel industry is not sunrise industry, the company's growth is not high; while some small and medium sized companies although growth is high, but the performance is poor, the high priceearnings ratio, and thus to be able to choose a good stock, you need to make investment decisions among these goals a balanced approach that more than a numerical target in a given region of the optimization problem is known as multiobjective optimization.

In order to solve multi-objective optimization problem, we need to create a general mathematical model, "we must first determine its decision variables, the general case, the decision variables $n$ dimensional Euclidean space as a point $E^{n}$, namely:

$$
x=\left(x_{1}, x_{2}, x_{3} \cdots x_{n}\right) \in E^{n}
$$

Second is the objective function, in general it can be assumed with $p$ objective functions and decision variables are all about function, namely:

$$
f(x)=\left[f_{1}(x), f_{2}(x), \cdots f_{p}(x)\right]^{T}
$$

Finally, its constraints, from a mathematical point of view, there are two constraints: inequality constraints and equality constraints, constraints can be defined as the $\mathrm{m}$ inequality constraints and $\mathrm{k}$ equality constraints:

$$
\begin{cases}g_{i}(x) \leq 0 & i=1,2,3 \cdots m \\ h_{j}(x)=0 & j=1,2,3 \cdots k\end{cases}
$$

If all are the minimization of the objective function value, the multi-objective optimization problem can be described as the following mathematical model:

$$
\left\{\begin{array}{l}
\min f(x)=\left[f_{1}(x), f_{2}(x), \cdots f_{p}(x)\right]^{T} \\
x_{i}^{\alpha} \leq x_{i} \leq x_{i}^{\beta}
\end{array}\right.
$$

Where, $x$ is the decision variable, $f(x)$ is the objective function, $X$ represents the decision vector formed by the decision space $x, g_{i}(x)$ and $h_{j}(x)$ constraints $x$ feasible decision variables to determine the range, min represents Vector Minimization, namely vector target $f(x)=\left[f_{1}(x), f_{2}(x), \cdots f_{p}(x)\right]^{T}$ in certain constraints as far as possible the various sub-objective function minimization. It can be seen when the $p=1$, the mathematical model for a single objective optimization problem mathematical model. 


\subsection{Basic Definition of Multi-Objective Optimization}

Multi-objective optimization problem is that people in the production or frequently encountered problems in life, in most cases, due to multi-objective optimization problem in all its goals are in conflict, a sub-target improvement may cause the performance of other sub-goals reduced, in order to make optimal multiple targets simultaneously is impossible, and thus in solving multi-objective optimization problem for each sub-goal can only be coordinated and compromise treatment, so that each sub-objective functions are optimal as possible multiobjective optimization problem with a single objective optimization problem is essentially different, in order to properly solve multi-objective optimization problem the optimal solution, we must first multi-objective optimization of the basic concepts of a systematic exposition.

Definition 1: N Viola Space:

$$
\left\{\begin{array}{l}
x=\left(x_{1}, x_{2}, x_{3} \cdots x_{n}\right)^{T} \\
y=\left(y_{1}, y_{2}, y_{3} \cdots y_{n}\right)^{T} \\
x=y \text { Iff } x_{i}=y_{i} \quad \forall i=1,2,3 \cdots n \\
x>y \text { Iff } x_{i}>y_{i} \quad \forall i=1,2,3 \cdots n
\end{array}\right.
$$

Definition 2: Let $X \subseteq R^{m}$ is a multi-objective optimization model of the constraint set, $f(x) \in R^{p}$ is a vector objective function, $x_{1} \in X \quad x_{2} \in X$, (a) $f_{k}\left(x_{1}\right)<f_{k}\left(x_{2}\right)$ better solution called solution $x_{1}, x_{2}$.(b) $x_{1}$ weak solution of $f_{k}\left(x_{1}\right) \leq f_{k}\left(x_{2}\right)$ called superior solution $x_{2}$.(c) $f_{k}\left(x_{1}\right) \geq f_{k}\left(x_{2}\right)$ solution called indifference to solution $x_{1}, x_{2}$.

Definition 3: Let $X \subseteq R^{m}$ be a multi-objective optimization model constraint set, $f(x) \in R^{p}$ is a vector objective function, $x^{n} \in X$ and $x^{n}$ than the $X$ all the other points are superior, called $x^{n}$ is the multi-objective minimization model optimal solution.

By definition, multi-objective optimization problem is to make the optimal solution $\mathrm{x}$-vector objective function $f(x)$ for each sub-goal is to achieve the most advantages of the solution, obviously, in most cases, the optimal multi-objective optimization problem solution does not exist.

Definition 4: Pareto optimal solution: Let $X \subseteq R^{m}$ be a multi-objective optimization model constraint set, $f(x) \in R^{p}$ is the vector of the objective function. If $\xi \in X, \xi$ and there is no more than the superiority of $x$, then $\xi$ is a minimal model of multi-objective Pareto optimal solution, or non-inferior solution.

Definition 5: No inferior set with the front end: Let $X \subseteq R^{m}$ be a multi-objective optimization model constraint set, $f(x) \in R^{p}$ is a vector objective function. $\lambda \in X$ is a minimal model of multi-objective Pareto optimal solution set, then $\lambda$ is called noninferior set of $X, Y=f(\lambda)$ is called Pareto optimal front.

Seen from the above definition: (a) Multi-objective optimization problem with a single objective optimization problem is essentially different, in general, multiobjective optimization problem Pareto "optimal solution is a collection of the $\mathrm{Mu}$ most cases, similar to the single-objective optimization problem in a multi-objective optimal solution optimization problem does not exist, there is only Pareto optimal "multi-objective optimization problem is just a Pareto optimal solution acceptable" not bad "solution, and usually most multi-objective optimization problem with multiple Pareto optimal solution. (b) If a multi-objective optimization problem optimal solution exists, then the optimal solution must be Pareto optimal solution, and the Pareto optimal solution is also the optimal solution by only composed of 
these, do not contain other solutions, so can be so say, Pareto optimal solution is a multi-objective optimization problem reasonable solution set. (c) For practical application, must be based on the level of understanding of the problem and the decision-makers of personal preference, from a multi-objective optimization problem Pareto optimal solution set of one or more selected solution as a multiobjective optimization problem of optimal solution, so seeking more objective optimization problem the first step is to find all its Pareto optimal.

\subsection{Right Weight Coefficient of Genetic Algorithm}

Weight coefficient variation method is used to solve multi-objective optimization problem of the earliest methods. The basic idea is: For a multi-objective optimization problem, if for each of its sub-objective function $f_{i}(x)$ given different weights $w_{i}$, where the size of $w_{i}$ represents the corresponding sub-goals $f_{i}(x)$ in a multi-objective optimization problem in an important degree, the individual subgoals weighted linear function can be expressed as:

$$
\left\{\begin{array}{l}
f(x)=w_{1} f_{1}(x)+w_{2} f_{2}(x)+\cdots w_{p} f_{p}(x)=\sum_{i=1}^{p} w_{i} f_{i}(x) \\
w_{i}=\frac{\text { random }_{i}}{\text { random }_{1}+\text { random }_{2}+\cdots \text { random }_{p}}
\end{array}\right.
$$

As to the fitness function $\sum_{i=1}^{p} w_{i} f_{i}(x)$ roulette wheel selection can be determined by hybridization and mutation of the individual involved and so on. Thus, this method can provide a lot of random points to a valid interface search direction; this algorithm is used to Flow-shop scheduling problems, and achieved good results.

Here we have normalized the objective function, when the first $t$ Let $\left(x_{1}, x_{2}, x_{3} \cdots x_{p}\right)$ generation populations, so $h_{i}(t)=\max \left\{\left|f_{i}\left(x_{1}\right)\right|,\left|f_{i}\left(x_{2}\right)\right|,\left|f_{i}\left(x_{3}\right)\right| \cdots\left|f_{i}\left(x_{p}\right)\right|\right\}$, then $f_{i}(x)$ can be normalized to the new objective function:

$$
g_{i}(x)=\frac{f_{i}(x)}{h_{i}(t)}
$$

The new fitness function can be redefined as:

$$
G(x)=\sum_{i=1}^{m} w_{i} g_{i}(x)
$$

Adapted according to the size of the angle $g(x)$ with the roulette wheel selection operator $p_{0}(t)$ is selected from the initial population of parent and points $N$ for hybridization, parents set point set is $P$ :

$$
\left\{\begin{array}{l}
x_{k}^{i}=r \cdot x^{i}+(1-r) \cdot x^{i+1} \\
x_{k}^{i+1}=(1-r) \cdot x^{i}+r \cdot x^{i+1}
\end{array}\right.
$$

Wherein $r$ is a random number between $[0,1],\left[x^{i}, x^{i+1}\right] \in P,\left[x_{k}^{i}, x_{k}^{i+1}\right] \in p(t), p(t)$ is set after hybridization offspring.

Let $x=\left(x_{1}, x_{2}, x_{3} \cdots x_{n}\right)$ be a parent population of the body $p(t), j=1,2,3 \cdots n$, $x_{j}$ is $x \in p(t)$, page $j$ coordinate; $a_{j}<x_{j}<b_{j}, a_{j}$ and $b_{j}$, respectively, of the search points in the space coordinate $j$ The lower and upper bounds; $x_{j}^{k} x_{j}^{k} \in p(t)$ is the first coordinate $j, p(t)$ is the first step Offspring collection; $r$ is between $[0,1]$ random number between; $T$ is the maximum genetic algebra; $t$ for the current genetic algebra; $r(0,1)$ said that produce 0 or 1 random number. There are: 


$$
\begin{cases}x_{j}^{k}=x_{j}+\left(b_{j}-a_{j}\right) \cdot r \cdot\left(1-\frac{t}{T}\right) & \text { if } r(0,1)=0 \\ x_{j}^{k}=x_{j}-\left(b_{j}-a_{j}\right) \cdot r \cdot\left(1-\frac{t}{T}\right) & \text { if } r(0,1)=1\end{cases}
$$

Non-uniform mutation is to participate in the weight variation did a random disturbance; this disturbance in the early evolution of a relatively large range, but with the evolution of generation increases, changes in disturbance gradually decreases.

\section{System Assessments}

\subsection{Fitness Function and Operator}

Let $\left(x_{1}^{t}, x_{2}^{t}, x_{3}^{t} \cdots x_{N}^{t}\right)$ be the population in the first generation of the feasible solutions individual ${ }^{t}, N_{\text {is }}$ the population size, ${ }^{x_{i}^{t}}$ denotes the first-generation ${ }^{t} i$ individuals, $p_{i}^{t}$ is Pareto superior to individual $x_{i}^{t}$ population the number of individuals, defined as the first $s_{i}^{t}\left(s_{i}^{t}=p_{i}^{t}+1\right) t x_{i}^{t}$ behalf of individuals ordinal values, denoted $x_{i}^{t}$ individual fitness function $t$ to $f_{i}^{t}=\frac{1}{s_{i}^{t}}$ if the first generation of Pareto superior to the more individuals $t, x_{i}^{t}$ order value is larger, the lower the degree of its adaptation, ie individual $x_{i}^{t}$ worse quality; Contrary, adapt the higher the better the quality of the individual $x_{i}^{t}$.

By the uniform crossover design ideas, assume that hybridizes to two parent individuals were $p_{1}=\left(x_{1}, x_{2}, x_{3} \cdots x_{n}\right)^{T}, p_{2}=\left(y_{1}, y_{2}, y_{3} \cdots y_{n}\right)^{T}$ component of the inserted between their corresponding points, towered equally divided into $q-1$ :

$$
\left\{\begin{array}{l}
y_{1}=x_{1}, x_{1}+d, x_{1}+2 d, \cdots+x_{1}+(q-1) d \\
y_{2}=x_{2}, x_{2}+d, x_{2}+2 d, \cdots+x_{2}+(q-1) d \\
\cdots \\
y_{n}=x_{n}, x_{n}+d, x_{n}+2 d, \cdots+x_{n}+(q-1) d
\end{array}\right.
$$

Where $d=y_{i}-x_{i} / q-1$, is the length of the corresponding component of the aliquots, as the individual components of uniform design factor, $x_{i}+(q-1) d, i$ is the first horizontal factor of $q$, where $q$ and $n$ must be greater than a prime number. Uniform design method according to the previous chapter, $x$ and $y$ will be generated in the hypercube formed by the $q$ uniformly distributed points, we these points as $q, x$ and $y$ offspring generated after the hybridization. Since the plurality of offspring in its two parent hypercube formed uniformly distributed, which is equivalent in the two parent individuals do a local search around.

According to the crossover probability of individual populations to determine whether to participate in the cross, and then a random string of individual genes set a crossover point, at the intersection of genes on arithmetic crossover operation, matching the intersection in front of the individual genes directly copied to the offspring, after the intersection after the exchange of genes assigned to the corresponding offspring. Single-point crossover complex not only maintains a simulated binary crossover advantages, but also by increasing the solving arithmetic crossover search area, enhanced search capabilities of the algorithm. 


\subsection{Genetic Algorithm Design and Implementation}

(a) Encoding: binary encoding. (b) The initial population: For the generation of the initial population, using a random generator of. (c) Fitness function: fitness function using equation (8) in the design of the fitness function. (d) Selection operator: Use roulette wheel selection and elitist strategy of combining strategy with a parent in the fitness value of the top surface of $M$ individuals through crossover and mutation to replace the population after the fitness value of row $M$ individuals in the final, so that offspring and parent to participate in competition, in order to prevent destruction of crossover and mutation operations populations fine mode. (e) Crossover: Use a single-point crossover operator, in which the crossover probability using Equation (9) in the design. (f) Mutation Operator: Use basic bit mutation operator, mutation probability using Equation (10) in the design.

Step1: According to the size of the fitness value of all individuals in contemporary populations sorted and selected the top surface of $\mathrm{M}$ individuals;

Step2: Let contemporary populations all individuals are involved in crossover and mutation operations to produce the next generation population;

Step3: The next generation of all individuals in the population according to the size of fitness value sorted out at the back of the $M$ individuals;

Step4: Selected with step1 replaced step3 $\mathrm{M}$ individuals to find the $\mathrm{M}$ individuals, generate a new generation.

In the optimization process, the literature [12] Improved adaptive genetic algorithm crossover and mutation probability design; paper improved genetic algorithm crossover probability and mutation probability formula using the formula (8), the formula (9) in the design; join fitness function is designed in this paper an improved genetic algorithm crossover and mutation probability design, fitness function formula using the formula (10) in the design.

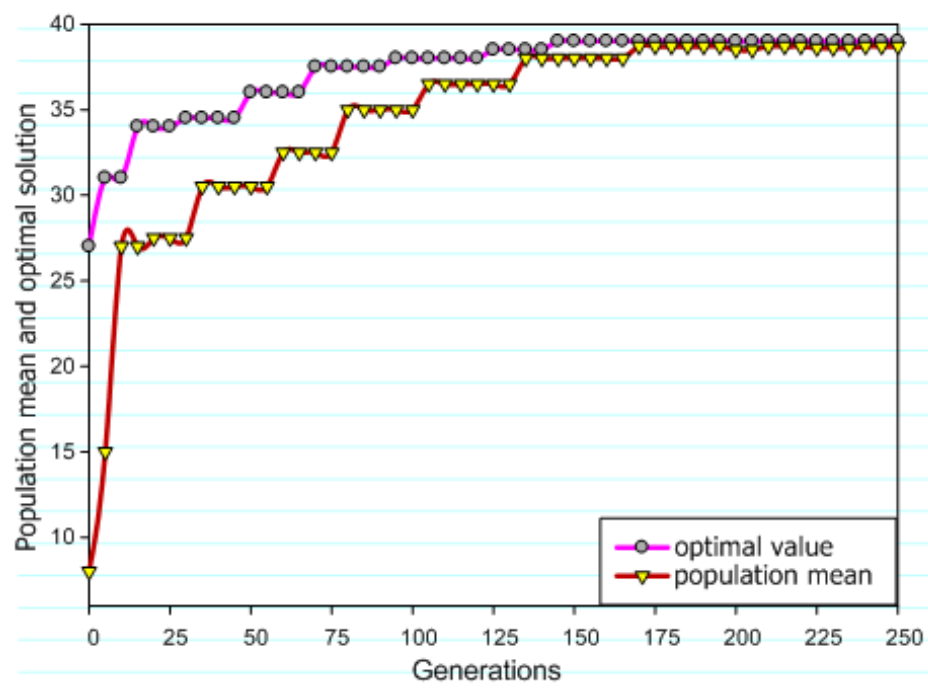

Figure 1. Population Changes in the Mean and the Optimal Value 


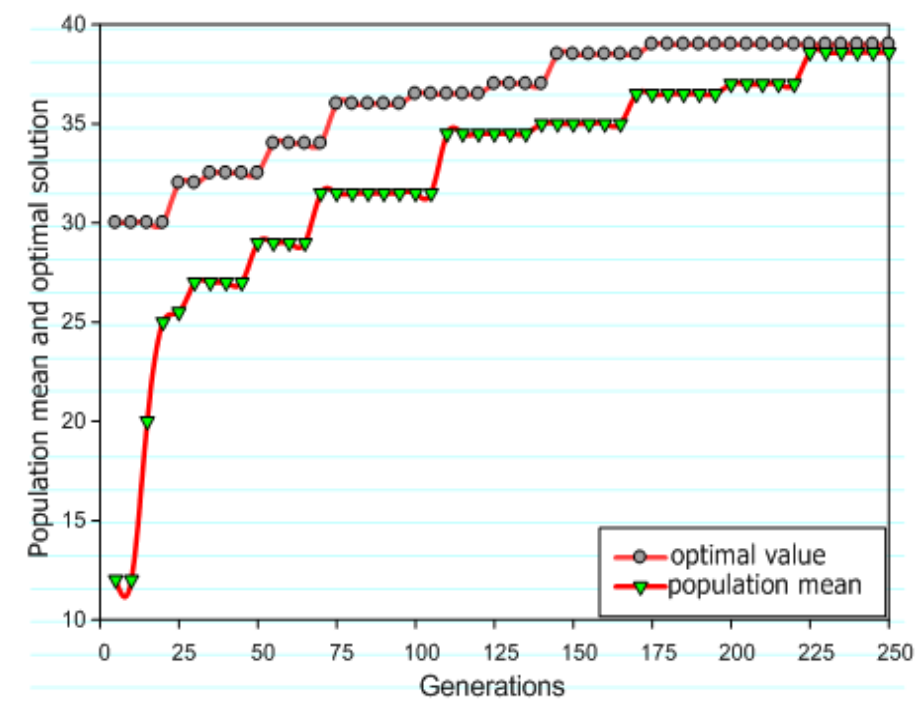

\section{Figure 2. IAGA Population Mean and the Optimal Value Changes}

By Figures 1 and 2 of the observation can be found using only this improved crossover and mutation probability formulas genetic algorithm IAGA average number of iterations than the literature [12] Improved adaptive genetic algorithm IAGA less; improved this article crossover probability and mutation probability formula and join the fitness function designed in this paper an improved genetic algorithm IAGA2 average number of iterations to be less than the algorithm IAGA1. Literature [12] Improved adaptive genetic algorithm IAGA average number of iterations is about IAGA2 2 times; adaptive genetic algorithm AGA average number of iterations is IAGA2 about four times. This article shows an improved genetic algorithm in computing speed is obviously better than the adaptive genetic algorithm and the literature [12] improved adaptive genetic algorithm.

\subsection{Application Example}

There are 20 rescue nodes, 8 shelters, 3 boat docking and 5 boats. Data in Table 1-4:

Table 1. Point of Rescues

\begin{tabular}{cccccc}
\hline NO. & $\begin{array}{c}\mathrm{X}- \\
\text { coordinate/km }\end{array}$ & $\begin{array}{c}\text { Y- } \\
\text { coordinate/km }\end{array}$ & $\begin{array}{c}\text { Number } \\
\text { of victim }\end{array}$ & $\begin{array}{c}\text { The time rescue } \\
\text { one person/min }\end{array}$ & $\begin{array}{c}\text { The last time } \\
\text { rescue } \\
\text { completed/h }\end{array}$ \\
\hline 1 & 3 & 42 & 3 & $0.78,0.90,1.08$ & 2 \\
2 & 16 & 48 & 2 & $0.78,0.90,1.08$ & 2 \\
3 & 19 & 69 & 36 & $0.78,0.90,1.08$ & 5 \\
4 & 37 & 53 & 5 & $0.78,0.90,1.08$ & 2 \\
5 & 49 & 64 & 5 & $0.78,0.90,1.08$ & 4 \\
6 & 55 & 79 & 5 & $0.78,0.90,1.08$ & 3.5 \\
7 & 47 & 87 & 4 & $0.78,0.90,1.08$ & 3 \\
8 & 40 & 89 & 9 & $0.78,0.90,1.08$ & 4 \\
9 & 41 & 9 & 2 & $0.78,0.90,1.08$ & 3.5 \\
10 & 47 & 3 & 8 & $0.78,0.90,1.08$ & 4 \\
11 & 45 & 16 & 25 & $0.48,0.60,0.78$ & 4 \\
12 & 54 & 3 & 9 & $0.48,0.60,0.78$ & 2 \\
13 & 55 & 21 & 3 & $0.48,0.60,0.78$ & 3 \\
\hline
\end{tabular}




\begin{tabular}{lllccc}
\hline 14 & 61 & 30 & 2 & $0.48,0.60,0.78$ & 3.5 \\
15 & 64 & 52 & 31 & $0.48,0.60,0.78$ & 4 \\
16 & 72 & 45 & 9 & $0.48,0.60,0.78$ & 5 \\
17 & 74 & 31 & 7 & $0.48,0.60,0.78$ & 4 \\
18 & 85 & 60 & 18 & $0.48,0.60,0.78$ & 5 \\
19 & 88 & 66 & 8 & $0.48,0.60,0.78$ & 2 \\
20 & 93 & 62 & 2 & $0.48,0.60,0.78$ & 3 \\
\hline
\end{tabular}

Table 2. Shelters

\begin{tabular}{cccc}
\hline NO & X-coordinate & Y-coordinate & Capacity \\
\hline 1 & 30 & 28 & 50 \\
2 & 75 & 80 & 50 \\
3 & 12 & 60 & 100 \\
4 & 38 & 77 & 100 \\
5 & 62 & 13 & 100 \\
6 & 68 & 33 & 50 \\
7 & 87 & 49 & 50 \\
8 & 95 & 97 & 50 \\
\hline
\end{tabular}

Table 3. Boat Docking

\begin{tabular}{ccc}
\hline NO & Coordinate & Boats \\
\hline 1 & $(30,62)$ & $1 、 2$ \\
2 & $(50,35)$ & $3 、 4$ \\
3 & $(71,69)$ & 5 \\
\hline
\end{tabular}

Table 4. Boats

\begin{tabular}{cccc}
\hline NO & Transport distance & Capacit & Speed km/h \\
\hline $1 、$ & 120 & 10 & 60 \\
$2 、$ & 100 & 20 & 50 \\
5 & 90 & 30 & 40 \\
\hline
\end{tabular}

The algorithm parameters are as follows: num $=100 ;$ MAXGEN $=300 ; \delta_{1}=0.9$; $\delta_{2}=1.2 ; \alpha=0.9 ; \beta=1 ; \omega=0.8 ; q=20 ;$ Crossover rate is 0.9 ; mutation rate is 0.05 .

The result is relatively stable: The ten times average target value is 35.2518 , the deviation to the average target value of the worst solution and the best solution is only $1.66 \%$ and $2.38 \%$. The best result was shown in Figure 1 . We no longer show the routes and every rescue nodes' completed time. There will be several completed time to one rescue node that indicate the node was not rescued by only one boat. 


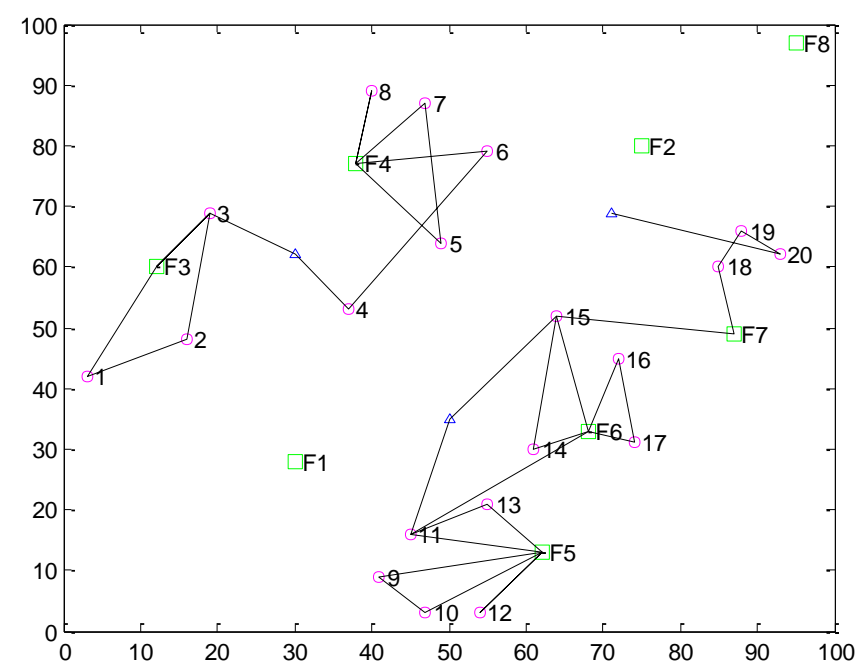

Figure 3. Calculation Results

\section{Conclusions}

The main task of multi-objective optimization is to improve the quality of the solution and maintain the solution of a broad distribution and uniformity [13], the weighted sum genetic algorithm is a straightforward, practical, strong multiobjective genetic algorithms. This paper focuses on the weighted sum of the genetic algorithm, uniform design created by combining the initial population, and their respective objective function Number of standardization, the establishment of a new fitness function, and proposes a dynamic allocation weighting scheme, designed a new weight-based allocation strategy Multi-objective Genetic Algorithm for multiobjective optimization problem. Second, the design of a uniform design method based on multi-objective optimization genetic algorithm, and gives a proof of convergence of the algorithm by simulation to verify the effectiveness of the algorithm.

\section{References}

[1] K. Deb, A. Pratap and S. Agarwal, "A Fat and Elitist Multi-objective Genetic Algorithm: NSGA- II", Evolutionary Computation, vol. 6, no. 2, (2002), pp. 182-197.

[2] B. William, "The papers of Benjamin Franklin", Yale University Press, New Haven, (1975).

[3] C. Koopmans, "Analysis of Production as an Efficient Combination of Activities", Activity Analysis of Production and Allocation, vol. 3, no. 2, (1951), pp. 33-97.

[4] R. Jin, LS Hong, C Wang, LF Wu and WC Si, "A Hierarchical Clustering Community Algorithm Which Missed the Signal in the Process of Transmission", International Information and Engineering Technology Association, vol. 2, no. 3, (2015), pp. 27-34.

[5] Y.Q. Yan, "A Practice Guide of Predicting Resource Consumption in a Web Servicer", International Information and Engineering Technology Association, vol. 2, no. 3, (2015), pp. 1-8.

[6] J. D. Sehaffer, "Multiple Objective Optimization with Vector Evaluated Genetic Algorithms", Proceedings of the 1st International Conference on Genetic Algorithms, Boston, USA, (1986), March 21-24.

[7] A. Carlos and M. Coello, "Evolutionary Multi-objective Optimization: Current and Future Challenges, Advances in Soft Computing Engineering", Design and Manufacturing, vol. 9, no. 6, (2013), pp. 243256.

[8] Y.P. Wang, Y.C. Jiao and F.S. Zhang, "Uniform and Orthogonal Genetic Algorithm for Multi-objective Optimization”, Journal of Systems Engineering, vol. 28, no. 6, (2012), pp. 481- 486.

[9] Z.H. Guan, J.S. Kou and M.Q. Li, “A Co-evolutionary Algorithm Based on $\varepsilon$-Constraint and Augmented Lagrangian Methods”, Systems Engineering and Electronics, vol. 34, no. 9, (2013), pp.V33-37. 
[10] G.M. Gong, L.C. Jiao, D.D. Yang and W.P. Ma, "Research on Evolutionary Multi-objective optimization Algorithm", Journal of Software, vol. 30, no. 2, (2012), pp. 271-289.

[11] J.H. Zheng, Z.Z. Shi and A. Xie, "A Fast Multi-Objective Genetic Algorithm Based on Clustering", Journal of Computer Research and Development, vol. 52, No. 7, (2011), pp. 1081-1087.

[12] J. Jin and Y. Su, "An Improved Adaptive Genetic Algorithm", Computer Engineering and applications, vol. 22, no. 11, (2013), pp. 3164-3169.

[13] H. Li and Z.Y. Chang, "Multi-objective Optimization Problem Based on Genetic Algorithm", Information Technology Journal, vol. 11, no.22, (2013), pp. 6968-6973.

\section{Authors}

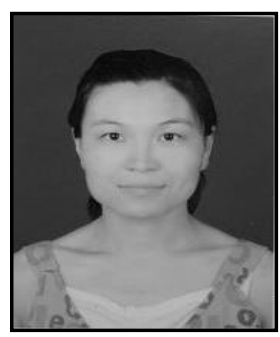

Yali Yun, she was born in 1975 in Luoyang city henan province, in 2004 graduated from Southeast University, Master of Science; She is a lecturer in College of Computer and Information Engineering, Luoyang Institute of Science and Technology. The main research interest is artificial intelligence, cloud computing.

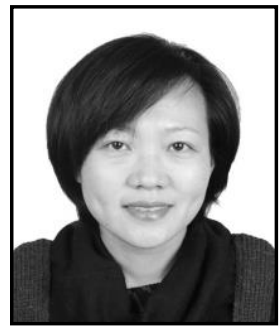

Yaping Li, she was born in 1968 in Kaifeng city Henan province. In 1990 graduated from Zhengzhou University of Computer \& Science Department, Master of Science; she is an associate professor in College of Computer and Information Engineering, Luoyang Institute of Science and Technology. The main research interest is wireless sensor network, parallel computing. 
International Journal of Hybrid Information Technology Vol. 9, No.7 (2016) 